\title{
Genome Instability as a Consequence of Defects in the Resolution of Recombination Intermediates
}

\author{
Stephen C. West and Ying Wai Chan \\ The Francis Crick Institute, London NW1 1AT, United Kingdom \\ Correspondence: stephen.west@crick.ac.uk
}

\begin{abstract}
The efficient processing of homologous recombination (HR) intermediates, which often contain four-way structures known as Holliday junctions (HJs), is required for proper chromosome segregation at mitosis. Eukaryotic cells possess three distinct pathways of resolution: (i) HJ dissolution mediated by BLM-topoisomerase III $\alpha$-RMI1-RMI2 (BTR) complex, and HJ resolution catalyzed by either (ii) SLX1-SLX4-MUS81-EME1-XPF-ERCC1 (SMX complex) or (iii) GEN1. The BTR pathway acts at all times throughout the cell cycle, whereas the actions of SMX and GEN1 are restrained in S phase and become elevated late in the cell cycle to ensure the resolution of persistent recombination intermediates before mitotic division. By developing a "resolvase-deficient" model system in which the activities of MUS81 and GEN1 are compromised, we have explored the fate of unresolved recombination intermediates. We find that covalently linked sister chromatids promote the formation of a new class of ultrafine bridges at anaphase that we term HR-UFBs. These bridges are broken at cell division, leading to activation of the DNA damage checkpoint and repair by nonhomologous end joining (NHEJ) in the next cell cycle. As a consequence, high levels of gross chromosomal rearrangements and aberrations are observed, together with frequent cell death. These results show that the $\mathrm{HJ}$ resolvases provide essential functions for the resolution of recombination intermediates, even in cells that remain proficient for BTR-mediated HJ dissolution.
\end{abstract}

Homologous recombination provides an essential mechanism for the repair of DNA breaks that arise from the demise of stalled replication forks or are induced by genotoxic agents. Recombination usually takes place between sister chromatids, as one sister provides a template for the error-free repair of the broken DNA. Joint molecule intermediates of homologous recombination often take the form of a four-way junction, also known as a Holliday junction ( $\mathrm{HJ})$, that needs to be resolved before chromosome segregation (Holliday 1964; West 2003; Wyatt and West 2014).

In recent years, there have been rapid developments in our understanding of the processes by which recombination intermediates, and in particular HJs, are processed. In contrast to simple organisms such as bacteria, which have a single HJ resolvase known as the RuvC protein (Dunderdale et al. 1991; Iwasaki et al. 1991; West 1997), eukaryotes have three distinct pathways that process junctions. These pathways use two distinct mechanisms: topoisomeraseinduced dissolution and nuclease-mediated resolution.

\section{MECHANISMS AND REGULATION OF RECOMBINATION INTERMEDIATE PROCESSING}

A four-subunit complex, containing BLM-topoisomerase III $\alpha$-RMI1-RMI2, known as the BTR complex, promotes the dissolution of double HJs. This complex promotes the convergent migration of two HJs to produce a hemicatenane structure that is dissolved by topoisomerase action (Wu and Hickson 2003). BTR-mediated HJ dissolution takes place throughout the cell cycle and gives rise exclusively to noncrossover products (Fig. 1). Cells derived from individuals with Bloom syndrome (BS), which is caused by mutations in BLM, show the diagnostic feature of a high frequency of sister chromatid exchanges and increased genome instability. Consequently, these individuals are predisposed to a broad spectrum of earlyonset cancers (Ray and German 1984; Hickson 2003).

Persistent double HJs, and single HJs that cannot serve as a substrate for BLM, are resolved by structure-selective nucleases (resolvases). These enzymes cut HJs by introducing coordinated nicks across the junction and give rise to nicked duplex products that are crossovers (COs) or noncrossovers (NCOs). The elevated frequency of sister chromatid exchanges (i.e., COs between sister chromatids) observed in BLM-deficient cells results from these resolution events (Wechsler et al. 2011).

There are two distinct nucleolytic pathways for resolution (Fig. 1). The first involves a complex that forms in a cell cycle-specific manner by interactions between MUS81-EME1 and a constitutive complex of SLX1SLX4-XPF-ERCC1 (Wyatt et al. 2017). The resulting complex, which we term SMX, comprises three nuclease activities: SLX1-SLX4, MUS81-EME1, and XPFERCC1 (Fig. 2). Direct interactions between SLX4 and MUS81 occur at prometaphase in response to CDK/ PLK1-mediated phosphorylation (Svendsen et al. 2009; Castor et al. 2013; Wyatt et al. 2013; Duda et al. 2016). The SMX complex resolves HJs by SLX1-mediated introduction of the first nick and by MUS81-mediated counternicking. Both incisions occur within the lifetime of the

(C) 2017 West and Chan. This article is distributed under the terms of the Creative Commons Attribution License, which permits unrestricted reuse and redistribution provided that the original author and source are credited. 


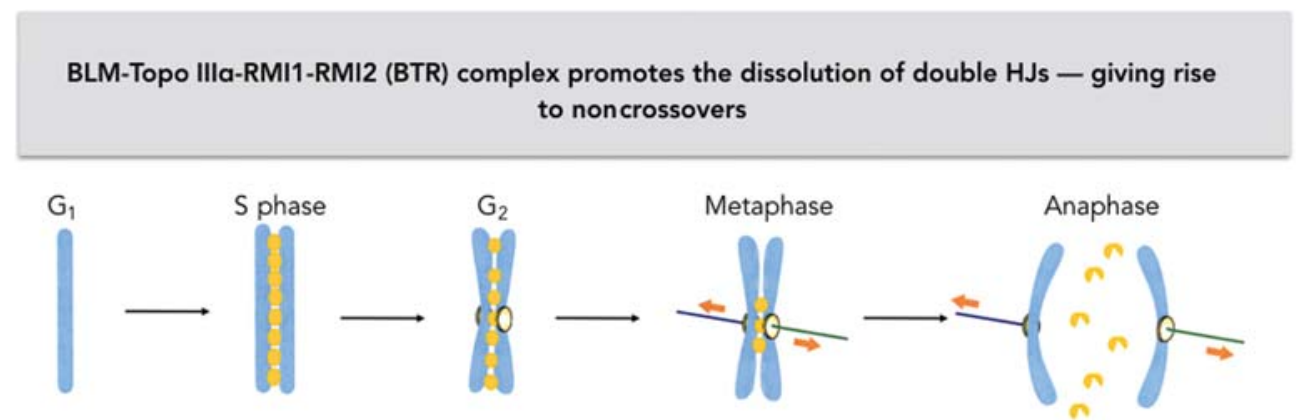

At $G_{2} / M$, MUS81-EME1 is activated by formation of the SMX trinuclease complex - acts upon single HJs, persistent double $\mathrm{HJs}$, and late RFs

Figure 1. Mechanisms for the processing of recombination intermediates in mitotic human cells. The BTR pathway is active throughout the cell cycle and promotes the dissolution of double Holliday junctions (HJs) to form noncrossover products. Later in the cell cycle, at prometaphase, MUS81-EME1 combines with the SLX1-SLX4-XPF-ERCC1 complex, in response to CDK/PLK1 phosphorylation events, to form the SMX trinuclease complex. SMX acts upon double HJs that have escaped the attention of BTR, single HJs that cannot be dissolved by BTR, and any late replication intermediates such as those present at common fragile sites. Finally, GEN1 protein, which is primarily cytoplasmic, gains access to any remaining recombination/replication intermediates upon breakdown of the nuclear envelope. SMX and GEN1 give rise to crossovers and noncrossovers.

SMX-HJ DNA complex (Wyatt et al. 2013, 2017). The interaction of MUS81-EME1 with SLX4 activates MUS81 for productive cleavage, in reactions that are thought to involve interplay between the amino-terminal HhH self-inhibitory domain of MUS81 and SLX4 (Wyatt et al. 2017). The nuclease activity of XPF is not required for cleavage, although XPF-ERCC1 may play a stimulatory role by contributing to the stability of the complex.

The second pathway of nucleolytic resolution involves the GEN1 HJ resolvase (Fig. 1). GEN1 shows nuclease activity on 5' flaps, replication fork structures, and HJs (Ip et al. 2008; Rass et al. 2010; Chan and West 2015; Bellendir et al. 2017). The mechanism of HJ cleavage by GEN1 is similar to that showed by the prototypic HJ resolvase RuvC, as symmetrical incisions are introduced across the junction point by the GEN1 homodimer. Like SMX, the actions of GEN1 are restricted to the late stages of the cell cycle, although in this case it is driven by nuclear exclusion (Chan and West 2014). Indeed, GEN1's HJ resolvase activity appears to be restrained until
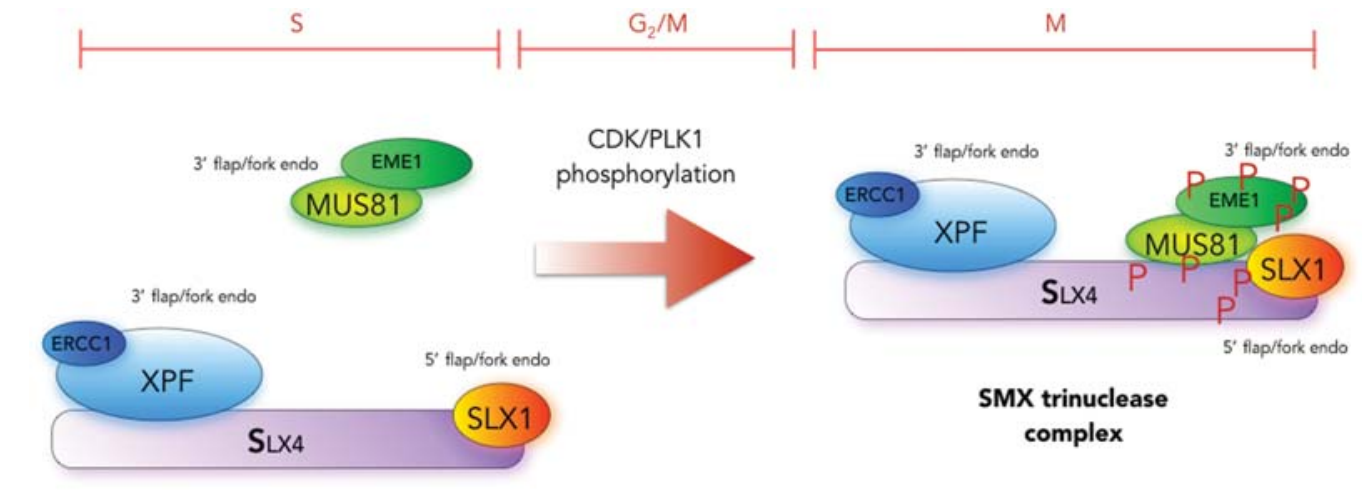

Figure 2. Cell cycle-mediated activation of MUS81-EME1 by formation of the SMX complex. The diagram indicates the interaction of MUS81-EME1 with the SLX1-SLX4-XPF-ERCC1 complex at prometaphase, in response to CDK/PLK1 phosphorylation. The resulting SLX1-SLX4-MUS81-EME1-XPF-ERCC1 (SMX) complex is capable of cleaving a range of replication and recombination intermediates. By association with SLX1-SLX4-XPF-ERCC1, the MUS81-EME1 nuclease is activated. 
nuclear membrane breakdown at mitosis. Once the nuclear membrane is dissolved, GEN1 will gain access to, and resolve, any persistent covalent bridges that link sister chromatids and so enable chromosome segregation.

\section{A MODEL SYSTEM FOR RESOLVASE DEFICIENCY}

The cellular importance of HJ processing in mammalian cells is apparent from the synthetic lethality observed in cells depleted for BLM (dissolution pathway) and SLX4 (resolution pathway) or SLX4 and GEN1 (both resolution pathways) (Garner et al. 2013; Wyatt et al. 2013; Sarbajna et al. 2014). This mortality is likely to stem from gross chromosomal abnormalities and mitotic defects (Wechsler et al. 2011; Garner et al. 2013; Wyatt et al. 2013; Sarbajna et al. 2014).

To develop a cellular system for the detailed analysis of resolvase deficiency, we recently used short interfering RNA (siRNA) to deplete MUS81 from a $G E N 1^{-/-}$cell line made using CRISPR (clustered regularly interspaced short palindromic repeat)-Cas9 (Chan and West 2015; Chan et al. 2017). Clonogenic survival assays showed that there was massive synthetic lethality, with $<10 \%$ survival, and that the cells were highly sensitive to treatment with DNA damaging agents such as cisplatin (Fig. 3A). Moreover, we observed that the metaphase chromosomes were elongated and showed indentations along their length (Fig. 3B) because of the presence of unresolved recombination intermediates (Wechsler et al. 2011; Chan et al. 2017). Similar defects have been observed in BLMdepleted SLX4 null cells and in cisplatin-treated cells depleted of SLX4 and GEN1 or MUS81 and GEN1 (Garner et al. 2013; Sarbajna et al. 2014). Segmentation occurred at equivalent positions on the two sister chromatids and was rescued by expression of a bacterial HJ resolvase such as RusA (Wechsler et al. 2011; Garner et al. 2013; Chan et al. 2017). Because the indentations are free of condensins (e.g., SMC2), we previously proposed that the persistent chromatid bridges cause defects in chromosome condensation rather than chromosome breakage (Wechsler et al. 2011).
A

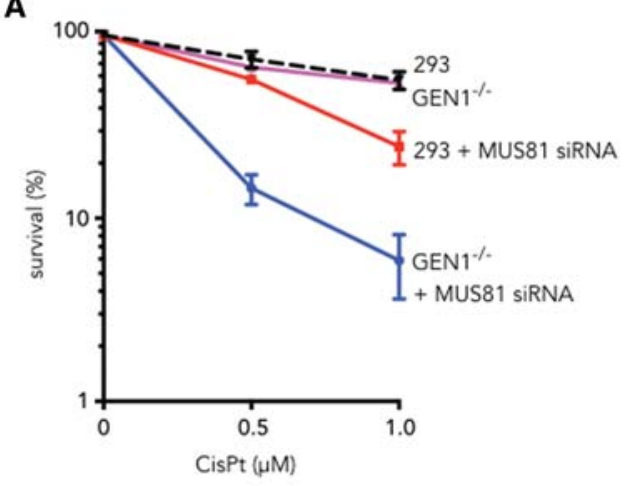

B

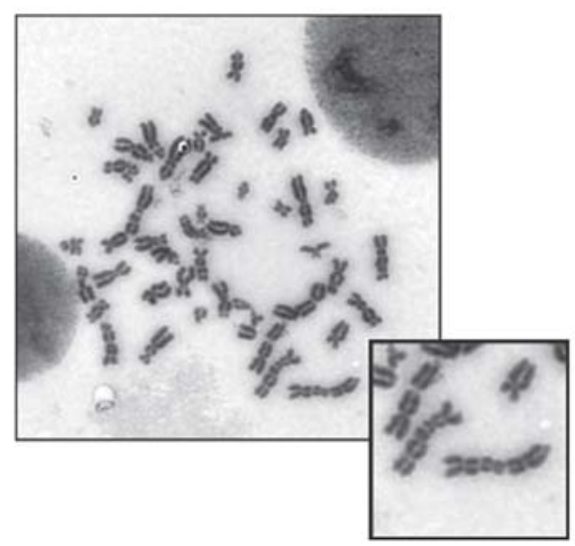

C
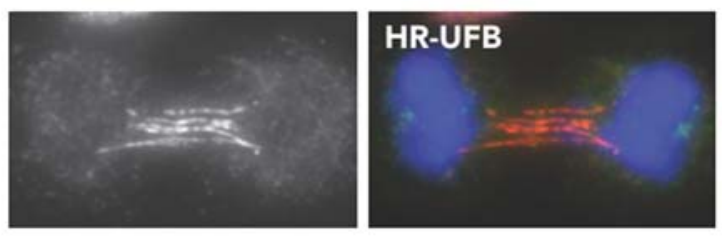

D
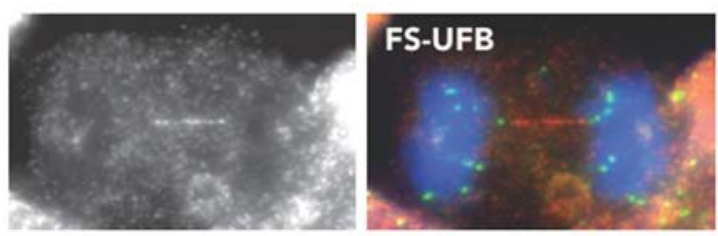

DAPI RPA FANCD2

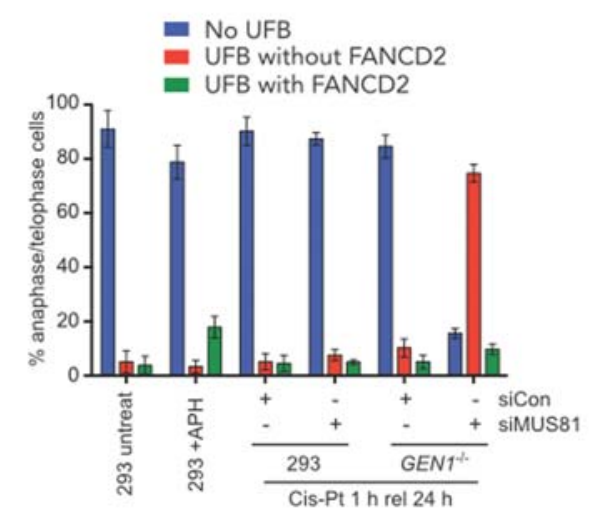

Figure 3. Phenotypic properties of resolvase-deficient cells. (A) Sensitivity of 293 and $G E N 1^{-/-}$cells treated with siRNA against MUS81, to cisplatin, measured by clonogenic survival. (B) Metaphase spreads from $G E N 1^{-1-}$ cells treated with siRNA against MUS81 and a brief cisplatin treatment reveal the presence of segmented chromosomes in which the sister chromatids remain interlinked. $(C)$ $G E N 1^{-/-}$cells treated with MUS81 siRNA and cisplatin form ultrafine bridges (UFBs) at anaphase. These are defined as homologous recombination UFBs (HR-UFBs). (D) 293 cells treated with aphidicolin, which causes mild replication stress, give rise to fragile siteassociated UFBs (FS-UFBs) which are distinguished from HR-UFBs by the presence of FANCD2 foci. RPA2, FANCD2, and DNAwere visualized using anti-RPA2 antibody (red), anti-FANCD2 antibody (green), and DAPI (blue). (E) Quantification of anaphase/telophase cells with RPA2-positive UFBs, with or without FANCD2 foci, as visualized in C. (Adapted from data in Chan et al. 2017.) 
A
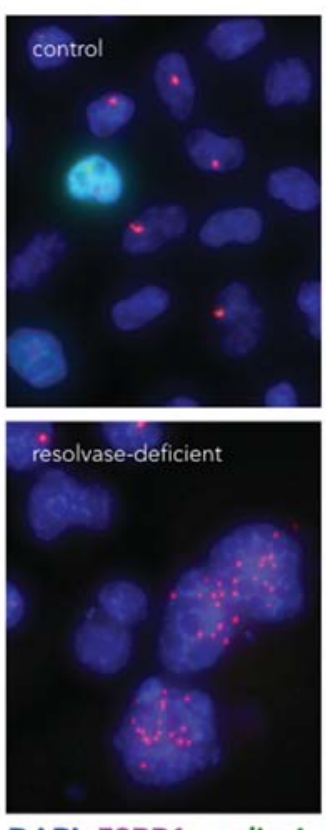

DAPI 53BP1 cyclin A
B
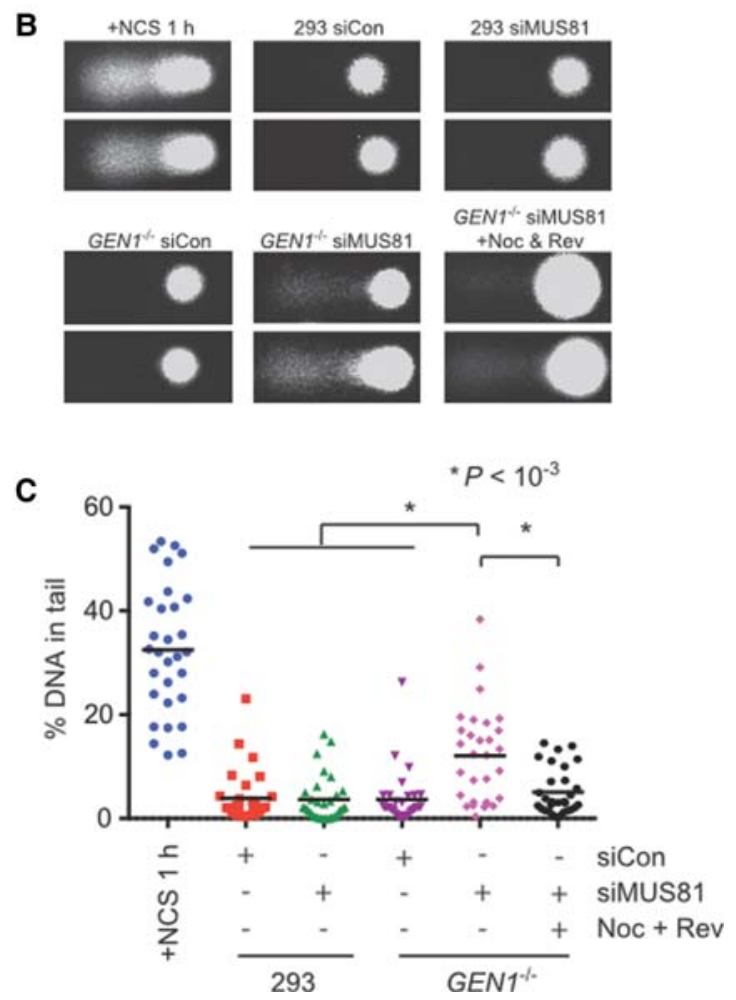

Figure 4. DNA breakage results from mitosis of resolvase-deficient cells. (A) 53BP1-positive nuclear bodies (red) in control, and SLX4 + GEN1-depleted, cisplatin-treated $\mathrm{G}_{1}$ (cyclin A-negative) HeLa cells. $(B, C) 293$ cells and $G E N 1^{-1-}$ cells were treated with control siRNA or siRNA against MUS81, as well as cisplatin. After further growth, cells were treated with or without nocodazole (Noc) and reversine (Rev). The DNA-PK inhibitor NU7026 was also added to the media. Cells were harvested after $20 \mathrm{~h}$ and DNA breaks were analyzed by comet assays. Neocarzinostatin-treated 293 cells were used as a control. (C) Quantification of the data shown in $B$. (Adapted from data presented in Sarbajna et al. 2014; Chan et al. 2017.)

\section{HR-UFBS LEAD TO CHROMOSOME ABERRATIONS}

Because unresolved recombination intermediates fail to elicit a checkpoint response, the resolvase-deficient cells enter mitosis with their sister chromatid bridges intact. As a consequence, we observe that $\sim 80 \%$ of the cells display replication protein A (RPA)-coated ultrafine bridges (UFBs) at anaphase (Fig. 3C,E; Chan et al. 2017). These UFBs, caused by unresolved homologous recombination (HR) intermediates (designated HR-UFBs), are distinct from replication stress-induced UFBs, which arise at common fragile sites (FS-UFBs) and are characterized by the presence of FANCD2 foci (Fig. 3D,E).

The presence of the single-strand binding protein RPA on the HR-UFBs indicates that the unresolved recombination intermediates are processed from duplex into singlestranded DNA. The PICH and BLM helicases were found to be essential for these processing reactions (Chan et al. 2017). Single-stranded UFBs are thought to be fragile enough to be broken by spindle forces at mitosis, and resolvase-deficient cells show high levels of 53BP1 (Sarbajna et al. 2014) or MDC1 (Chan et al. 2017) foci in the following $\mathrm{G}_{1}$ phase (Fig. $4 \mathrm{~A}$ ). These DNA damage signatures were not observed when cell division was blocked by treatment with nocodazole and reversine, which inhibit spindle assembly and the mitotic checkpoint, respectively. Moreover, direct evidence of DNA breaks was obtained using alkaline Comet assays (Fig. 4B,C), supporting the proposal that single stranded HR-UFBs are broken at mitotic division (Chan et al. 2017).

Coordinated with the presence of DNA breaks in $\mathrm{G}_{1}$ of the second cell cycle, we observed high levels of $\gamma \mathrm{H} 2 \mathrm{AX}$ and activation of ATM (ataxia telangiectasia mutated) at $\mathrm{G}_{2} / \mathrm{M}$, as measured by CHK2 and KAP1 phosphorylation (Chan et al. 2017). The cells then showed a cell cycle arrest. We did not find evidence of ATR (ataxia telangiectasia and Rad3-related) activation, indicating that the arrest was due to DNA breaks rather than activation of a replication checkpoint. Metaphase spreads prepared from the resolvase-deficient cells revealed a high frequency of end-toend chromosome fusions and radial chromosomes (Fig. 5A,B; Chan et al. 2017). When the cells were treated with the DNA-PKcs inhibitor NU7026, which inhibits nonhomologous end joining (NHEJ), we observed suppression of the fusion phenotype (Fig. 5C). These results show that the chromosome fusions and rearrangements are generated through the repair of breaks produced at the first mitotic division.

\section{CONCLUSION}

In summary, our work with this resolvase-deficient model cell system provides a clear picture of the way that unresolved recombination intermediates lead to cell 
A

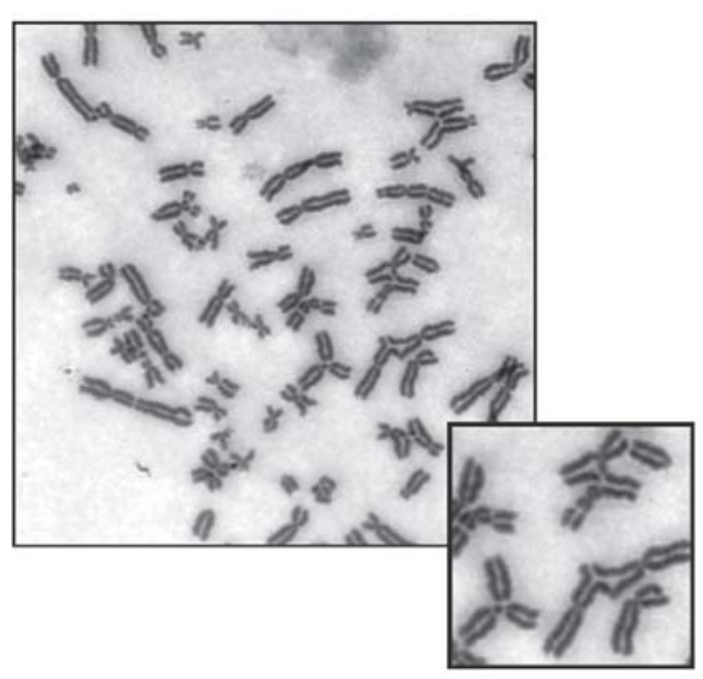

B

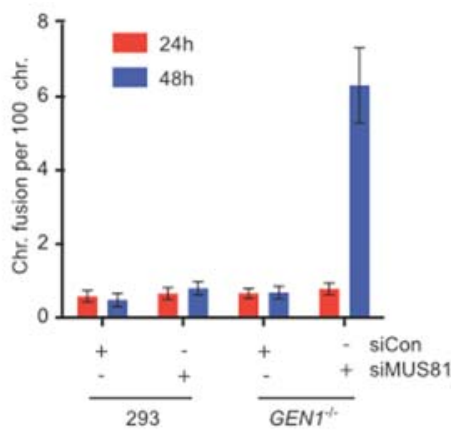

C

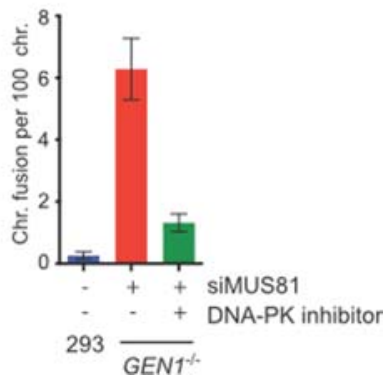

Figure 5. Gross chromosomal abnormalities result from DNA breakage. $(A)$ Representative image of a metaphase spread showing endto-end fusions and chromosome abnormalities in $G E N 1^{-1-}$ cells treated with MUS81 siRNA and cisplatin. (B) Quantification of fusions that arise in resolvase-deficient cells, as in $A$. $(C) \mathrm{GEN1}^{-1-}$ cells were treated as in $A$, except that the DNA-PK inhibitor NU7026 was added $24 \mathrm{~h}$ before harvest. Control siRNA-treated 293 cells were used as control. (Adapted from data presented in Chan et al. 2017.)

death. Importantly, the intermediates were found to persist until mitosis where they gave rise to UFBs at anaphase/ telophase. Subsequently, the interlinked sister chromatids were acted upon by PICH/BLM helicases, leading to their conversion into single-stranded RPA-coated UFBs. These HR-UFBs were distinct from replication-associated UFBs (FS-UFBs), which are characteristically flanked by the presence of FANCD2 foci. The single-stranded UFBs were then broken at mitotic division, thereby avoiding cytokinesis failure, but resulting in the presence of DNA breaks at $\mathrm{G}_{1}$ in the next cell cycle. These breaks were then repaired by NHEJ, resulting in gross chromosomal fusions and aberrations, leading to cell cycle arrest and cell death.

Although the resolvase-deficient cell system detailed here is somewhat artificial, as complete resolvase deficiency does not occur in nature, it provides a useful model that allows us to study the fate of unresolved recombination intermediates. Such intermediates may persist in highly replicating cancer cells in which the recombination "load" is often elevated compared to normal cells or in cells that have suffered DNA damage that increases the recombination frequency. Similarly, unresolved recombination intermediates may arise in cells that are compromised for their gatekeeper functions (i.e., HR vs. NHEJ) or those that express elevated levels of RAD51 (Xia et al. 1997; Klein 2008). A second important aspect of the work demonstrates that the resolvases do not simply provide a backup for the BTR pathway, because resolvase-deficient cells are effectively inviable. Indeed, they are essential factors that play a critical role in chromosome segregation.

\section{ACKNOWLEDGMENTS}

Work in the West laboratory at the Francis Crick Institute is supported by Cancer Research UK, the Medical Research Council, the Wellcome Trust, the European Research Council, and the Louis-Jeantet Foundation.

\section{REFERENCES}

Bellendir SP, Rognstad DJ, Morris LP, Zapotoczny G, Walton WG, Redinbo MR, Ramsden DA, Sekelsky J, Erie DA. 2017. Substrate preference of GEN endonucleases highlights the importance of branched structures as DNA damage repair intermediates. Nucleic Acids Res 45: 5333-5348.

Castor D, Nair N, Déclais AC, Lachaud C, Toth R, Macartney TJ, Lilley DMJ, Arthur JS, Rouse J. 2013. Cooperative control of Holliday junction resolution and DNA repair by the SLX1 and MUS81-EME1 nucleases. Mol Cell 52: 221-233.

Chan YW, West SC. 2014. Spatial control of the GEN1 Holliday junction resolvase ensures genome stability. Nat Commun 5: 4844.

Chan YW, West SC. 2015. GEN1 promotes Holliday junction resolution by a coordinated nick and counter-nick mechanism. Nucleic Acids Res 43: 10882-10892.

Chan YW, Fugger K, West SC. 2017. Unresolved recombination intermediates lead to ultra-fine bridges, chromosome breaks and aberrations. Nat Cell Biol doi: 10.1058/s41556-0170011-1.

Duda H, Arter M, Gloggnitzer J, Teloni F, Wild P, Blanco MG, Altmeyer M, Matos J. 2016. A mechanism for controlled breakage of under-replicated chromosomes during mitosis. Dev Cell 39: 740-755.

Dunderdale HJ, Benson FE, Parsons CA, Sharples GJ, Lloyd RG, West SC. 1991. Formation and resolution of recombina- 
tion intermediates by E. coli $\mathrm{RecA}$ and RuvC proteins. Nature 354: 506-510.

Garner E, Kim Y, Lach FP, Kottemann MC, Smogorzewska A. 2013. Human GEN1 and the SLX4-associated nucleases MUS81 and SLX1 are essential for the resolution of replication-induced Holliday junctions. Cell Rep 5: 207-215.

Hickson ID. 2003. RecQ helicase: Caretakers of the genome. Nat Rev Mol Cell Biol 3: 169-178.

Holliday R. 1964. A mechanism for gene conversion in fungi. Genet Res 89: 285-307.

Ip SCY, Rass U, Blanco MG, Flynn HR, Skehel JM, West SC. 2008. Identification of Holliday junction resolvases from humans and yeast. Nature 456: 357-361.

Iwasaki H, Takahagi M, Shiba T, Nakata A, Shinagawa H. 1991. Escherichia coli RuvC protein is an endonuclease that resolves the Holliday structure. EMBO J 10: 4381-4389.

Klein HL. 2008. The consequences of RAD51 overexpression for normal and tumor cells. DNA Repair 7: 686-693.

Rass U, Compton SA, Matos J, Singleton MR, Ip SCY, Blanco MG, Griffith JD, West SC. 2010. Mechanism of Holliday junction resolution by the human GEN1 protein. Genes Dev 24: 1559-1569.

Ray JH, German J. 1984. Bloom's syndrome and EM9 cells in BrdU-containing medium exhibit similarly elevated frequencies of sister chromatid exchange but dissimilar amounts of cellular proliferation and chromosome disruption. Chromosoma 90: 383-388.
Sarbajna S, Davies D, West SC. 2014. Roles of SLX1-SLX4, MUS81-EME1 and GEN1 in avoiding genome instability and mitotic catastrophe. Genes Dev 28: 1124-1136.

Svendsen JM, Smogorzewska A, Sowa ME, O'Connell BC, Gygi SP, Elledge SJ, Harper JW. 2009. Mammalian BTBD12/SLX4 assembles a Holliday junction resolvase and is required for DNA repair. Cell 138: 63-77.

Wechsler T, Newman S, West SC. 2011. Aberrant chromosome morphology in human cells defective for Holliday junction resolution. Nature 471: 642-646.

West SC. 1997. Processing of recombination intermediates by the RuvABC proteins. Annu Rev Genet 31: 213-244.

West SC. 2003. Molecular views of recombination proteins and their control. Nat Rev Mol Cell Biol 4: 435-445.

Wu L, Hickson ID. 2003. The Bloom's syndrome helicase suppresses crossing over during homologous recombination. $\mathrm{Na}$ ture 426: 870-874.

Wyatt HDM, West SC. 2014. Holliday junction resolvases. Cold Spring Harb Perspect Biol 6: a023192.

Wyatt HDM, Sarbajna S, Matos J, West SC. 2013. Coordinated actions of SLX1-SLX4 and MUS81-EME1 for Holliday junction resolution in human cells. Mol Cell 52: 234-247.

Wyatt HDM, Laister RC, Martin SR, Arrowsmith CH, West SC. 2017. The SMX DNA repair tri-nuclease. Mol Cell 65: 848-860.

Xia SJJ, Shammas MA, Reis RJS. 1997. Elevated recombination in immortal human cells is mediated by $h s$ RAD51 recombinase. Mol Cell Biol 17: 7151-7158. 


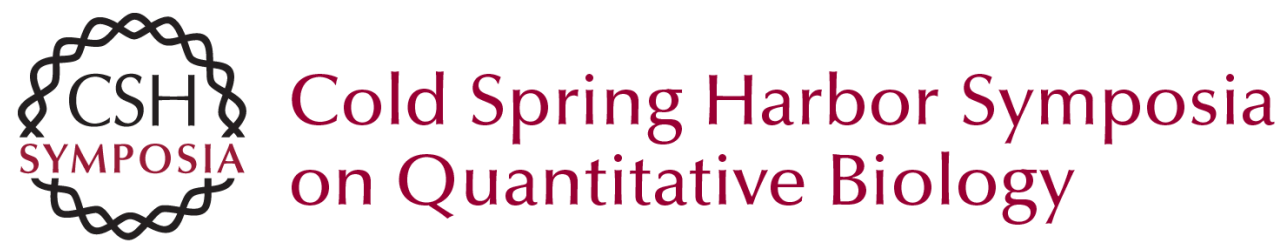

\section{Genome Instability as a Consequence of Defects in the Resolution of Recombination Intermediates}

Stephen C. West and Ying Wai Chan

Cold Spring Harb Symp Quant Biol 2017 82: 207-212 originally published online January 18, 2018

Access the most recent version at doi:10.1101/sqb.2017.82.034256

References This article cites 25 articles, 4 of which can be accessed free at: http://symposium.cshlp.org/content/82/207.full.html\#ref-list-1

Creative This article is distributed under the terms of the

Commons http://creativecommons.org/licenses/by/4.0/, which permits unrestricted

License reuse and redistribution provided that the original author and source are credited.

Email Alerting Receive free email alerts when new articles cite this article - sign up in Service the box at the top right corner of the article or click here. 